\title{
Follow-up guidelines after radical or partial nephrectomy for localized and locally advanced renal cell carcinoma
}

\author{
Wassim Kassouf, MD, FRCSC; ${ }^{*}$ Robert Siemens, MD, FRCSC; ${ }^{+}$ \\ Christopher Morash, MD, FRCSC;, Louis Lacombe, MD, FRCSC;; Michael Jewett, MD, FRCSC;"; \\ Larry Goldenberg, MD, FRCSC, FACS; ${ }^{* *}$ Joseph Chin, MD, FRCSC; ;t \\ Michael Chetner, MD, FRCSC; ;\# Christopher G. Wood, MD, FACS;;SS \\ Simon Tanguay, MD, FRCSC; ${ }^{*}$ Armen G. Aprikian, MD, FRCSC ${ }^{\text {III }}$
}

Can Urol Assoc J 2009;3(1):73-6

\section{Introduction and objectives}

Renal cell carcinoma (RCC) comprises approximately $2 \%$ of all malignancies. It is the seventh most common cancer and tenth most common cause of cancer-related deaths among men. ${ }^{1}$ Risk factors for RCC include smoking, obesity, and hereditary conditions associated with a mutation in the von-Hippel-Lindau gene (level 2). ${ }^{2,3}$ Surgical resection (radical or partial nephrectomy) remains the only effective therapy for clinically localized RCC. Publications that address surveillance after surgical extirpation are based on retrospective analysis, including some larger multicentre studies and well-designed controlled studies. ${ }^{4}$ Randomized prospective studies are sparse, rendering it difficult to obtain qualified evidence-based data. Although there is no clear consensus on surveillance after surgical extirpation for patients with RCC, this document attempts to provide some clarity and guidance for the practising urologist based on the current literature. Where possible, levels of evidence and grades of recommendations are provided employing the modified Oxford Centre for Evidence-based Medicine scheme.

\section{Rationale for surveillance}

Surveillance after surgery allows the urologist to monitor for postoperative complications, renal function, local recurrence, recurrence in the contralateral kidney and development of metastasis. Renal function and postoperative complications are commonly assessed by history, physical examination, and measurement of serum creatinine and hemoglobin at 4-6 weeks postsurgery. Long-term monitoring of serum creatinine is recommended particularly in patients with compromised renal function prior to surgery or significant increase in creatinine after surgery (Grade B). ${ }^{5}$ Early diagnosis of local and contralateral kidney recurrence (incidence $<2 \%$ ) is useful since the most effective treatment is surgical resection (level 3). ${ }^{6-8}$ Recurrence in the kidney is associated with positive surgical margins, multifocality and grade. Tumours that develop in the contralateral kidney can be treated with nephron-sparing surgery when detected at a small size. Patients who underwent surgery when local recurrences became symptomatic have a higher rate of incomplete resection of recurrence, positive surgical margins and poorer survival. ${ }^{9}$ Extensive tumour recurrence reduces the possibility of complete surgical resection, which is standard therapy for patients with local recurrence or resectable solitary metastasis (level 2). Furthermore, an early diagnosis of disease relapse may enhance efficacy of systemic therapy if the tumour burden is low. Hence, this supports the rationale for surveillance of patients to detect recurrences and metastases early (Grade B).

\section{Prognostic variables}

Predictors of disease relapse after surgical extirpation include RCC subtypes, tumour grade, local extent of the primary tumour, and presence of nodal metastasis (level 2)..$^{10}$ As such, these variables should be noted because they contribute important prognostic information. RCC with collecting duct carcinoma, medullary carcinoma and tumour with elements of sarcomatoid dedifferentiation exhibit higher metastatic potential. ${ }^{11,12}$ Localized chromophobe and papillary RCC portend a better prognosis. Other potential risk factors include performance status 
(ECOG), the presence of symptoms (localized or systemic), cachexia, anemia, platelet count, and primary tumour characteristics (tumour size, histologic coagulative necrosis, DNA ploidy) (level 3)..$^{13-16}$ Molecular markers including carbonic anhydrase IX, vascular endothelial growth factor, hypoxia inducible factor, Ki67, p53, PTEN, E-cadherins and others have demonstrated potential utility as prognostic markers (level 3). No molecular marker is currently recommended for utilization in the routine clinical setting. Several prognostic models have been published and externally validated. ${ }^{13,15,17}$ However, more extensive analysis in prospective randomized controlled studies are needed to prove superiority over the stage-specific strategy. The use of integrated prognostic system or nomograms is not routinely recommended although these tools may be useful for enrolling patients in clinical trials.

\section{Surveillance}

The intensity of radiological surveillance for patients will vary depending on the risk of devel- oping recurrence or metastases. Intensity and type of surveillance will be tailored according to a riskadapted approach. Most contemporary surveillance protocols have been formed on stage-based stratifications (Fig. 1). The Canadian guidelines for surveillance after nephrectomy for nonmetastatic renal cell carcinoma will be based on pathologic stage. Since the other previously mentioned factors have been shown to be prognostic mostly in single centre institutions, the incorporation of such factors to help tailor patients to more or less stringent follow-up protocols will be at the discretion of the treating physician (Grade C).

In the absence of randomized studies, conclusions will be based on large nonrandomized cohorts with long-term follow-up (level 3). To evaluate recurrence in the lung, routine chest $x$ ray is recommended. CT of the chest may be performed instead; however there is insufficient evidence to suggest a benefit in this setting (Grade C). To evaluate abdominal recurrences, $\mathrm{CT}$ of the abdomen is recommended, particularly in cases of tumour-associated symptoms; an abdominal

\begin{tabular}{|c|c|c|c|c|c|c|c|c|c|c|}
\hline & & & & \multicolumn{7}{|c|}{ Figure 1. Months Post-op } \\
\hline & 3 & 6 & 12 & 18 & 24 & 30 & 36 & 48 & 60 & 72 \\
\hline \multicolumn{11}{|l|}{ pT1 } \\
\hline \multicolumn{2}{|l|}{$\mathrm{Hx} \& \mathrm{PE}$} & & \multicolumn{2}{|l|}{$x$} & \multicolumn{2}{|l|}{$x$} & $x$ & $x$ & $x$ & $x$ \\
\hline Blood test & & & \multicolumn{2}{|l|}{$x$} & \multicolumn{2}{|l|}{$x$} & $x$ & $x$ & $x$ & $x$ \\
\hline CXR & & & \multicolumn{2}{|l|}{$x$} & \multicolumn{2}{|l|}{$x$} & $x$ & $x$ & $x$ & $x$ \\
\hline $\mathrm{CT}$ or U/S abd & & & & & \multicolumn{3}{|l|}{$x$} & & \multicolumn{2}{|l|}{$x$} \\
\hline \multicolumn{11}{|l|}{ pT2 } \\
\hline$H x \& P E$ & & $x$ & $x$ & $x$ & $x$ & $x$ & $x$ & $x$ & $x$ & $x$ \\
\hline Blood test & & $x$ & $x$ & $x$ & $x$ & $x$ & $x$ & $x$ & $x$ & $x$ \\
\hline CXR & & $x$ & $x$ & $x$ & \multirow[t]{2}{*}{$x$} & \multirow[t]{2}{*}{$x$} & $x$ & \multirow[t]{2}{*}{$x$} & $x$ & \multirow[t]{2}{*}{$x$} \\
\hline CT or U/S abd & & & \multicolumn{2}{|l|}{$x$} & & & $x$ & & $x$ & \\
\hline \multicolumn{11}{|l|}{ pT3 } \\
\hline$H x \& P E$ & & $x$ & $x$ & $x$ & $x$ & $x$ & $x$ & $\mathrm{x}$ & $x$ & $x$ \\
\hline Blood test & & $x$ & $x$ & $x$ & $x$ & $x$ & $x$ & $x$ & $x$ & $x$ \\
\hline CXR & & $x$ & $x$ & $x$ & $x$ & $x$ & $x$ & $x$ & $x$ & $x$ \\
\hline CT abd & & $x$ & $x$ & $x$ & $x$ & & $x$ & & $x$ & \\
\hline \multicolumn{11}{|l|}{ pTxN+ } \\
\hline$H x \& P E$ & $x$ & $x$ & $x$ & $x$ & $x$ & $x$ & $x$ & $x$ & $x$ & $x$ \\
\hline Blood test & $x$ & $x$ & $x$ & $x$ & $x$ & $x$ & $x$ & $x$ & $x$ & $x$ \\
\hline CXR & $x$ & $x$ & $x$ & $x$ & $x$ & $x$ & $x$ & $\mathrm{x}$ & $x$ & $x$ \\
\hline CT abd & $x$ & $x$ & $x$ & $x$ & $x$ & $x$ & $x$ & $x$ & $x$ & $x$ \\
\hline \multicolumn{11}{|c|}{$\begin{array}{l}\text { Hx \& PE: history and physical examination } \\
\text { Blood test: include complete blood count, serum chemistries, and liver function tests } \\
\text { CXR: can be alternated with chest CT } \\
\text { CT abd: can be alternated with abdominal ultrasound in pT1-2N0 patients } \\
\text { * -if patient is symptomatic or abnormal blood test, earlier radiologic investigations may be indicated } \\
\text {-follow-up beyond } 72 \text { months, refer to text for more details }\end{array}$} \\
\hline
\end{tabular}


ultrasound may be performed for lower risk patients (pT1 and pT2) (Grade C). CT head or bone scan is not routinely recommended unless clinically indicated.

\section{Recurrence patterns for pTl tumours}

Among several series, the local recurrence for pT1 lesions is very low $(<2 \%)$. Levy and colleagues ${ }^{9}$ reported $7 \%$ recurrence rate at median follow-up of 39 months with a median time to recurrence of 38 months (range 18-67 mo). Majority of recurrences were in the lung and no recurrences were found in the abdomen. None of the pulmonary recurrences were symptomatic. Similarly, the Canadian group has shown that median time to recurrence was 35 months (range 2-93 mo) and only $0.9 \%$ of patients recurred at 13,66 , and 93 months in the renal fossa and were asymptomatic. ${ }^{18}$ Several other reports showed similar findings. . $12,19-21$ Among several studies regarding RCC surveillance, the latest postnephrectomy recurrence in the lungs, abdomen, and bone was 67, 97, and 144 months, respectively..$^{9,12,18,20}$ Recommended surveillance will include clinical assessment, blood biochemistry, and chest $\mathrm{x}$-ray every year. Abdominal CT recommended at 24 and 60 months (Grade C). Follow-up is the same for partial nephrectomy for $<4 \mathrm{~cm}$ lesions since the local recurrence rates in this population is similar to radical nephrectomy (Grade B). CT abdomen at 3 months postoperative for patients treated with partial nephrectomy to evaluate the residual renal appearance is optional (Grade D). Annual abdominal ultrasound for patients post-partial nephrectomy is optional (Grade D).

\section{Recurrence patterns for pT2 tumours}

Several series have reported recurrences after a median time of 25-32 months., ${ }^{9,12,18,20}$ Levy and colleagues reported $27 \%$ recurrence rate at a median follow-up of 53 months with a time to recurrence of 32 months (range 3-115 mo). Only 2 patients developed metastasis within 6 months postoperative. The Canadian group reported a median time to recurrence of 25 months (range 3-95 mo) and 50\% were asymptomatic. ${ }^{18}$ Among several studies regarding RCC surveillance, the latest postnephrectomy recurrence in the lungs, abdomen, and bone was 96, 92, and 144 months, respectively. $9,12,18,20$ Recommended surveillance will include clinical assessment, blood biochemistry, and chest $\mathrm{x}$-ray every 6 months for 3 years then yearly. Abdominal CT recommended at 12, 36, 60, 84, and 108 months (Grade C).

\section{Recurrence patterns for $\mathrm{pT} 3$ tumours}

Levy and colleagues reported 39\% recurrence rate at a median follow-up of 31 months with a median time to recurrence of 17 months (range 2-88 mo). Stephenson and colleagues reported a median time to relapse of 14 months and 9 months for pT3a and pT3b tumours. ${ }^{18}$ Among several studies regarding RCC surveillance, the latest postnephrectomy recurrence in the lungs, abdomen, and bone was 138, 79 , and 65 months, respectively. ${ }^{9,12,18,20}$ Recommended surveillance will include clinical assessment, blood biochemistry, and chest $\mathrm{x}$-ray every 6 months for 3 years then yearly. Abdominal CT recommended at $6,12,18,24,36,60$ months then every 2 years (Grade C).

\section{Recurrence patterns of $\mathrm{pTxN}+$ tumours}

Canfield and colleagues reported a $70 \%$ recurrence rate at a median follow-up of 17.7 months with a median time to recurrence of 4.9 months..$^{22}$ Saidy and colleagues ${ }^{23}$ reported a $64 \%$ recurrence rate at a mean follow-up of 39 months with a median time to recurrence of 9 months. Most disease will recur within the first year and $>90 \%$ by 3 years. Most common sites of recurrences in order of decreasing frequency were retroperitoneal lymph nodes, lung, liver, bone, renal fossa, pelvis, and brain. Similar results are shown by the UCLA group. ${ }^{24}$ Recommended surveillance will include clinical assessment, blood biochemistry, chest x-ray, and $\mathrm{CT}$ of the abdomen/pelvis at 3 and 6 months, then every 6 months for 3 years then yearly (Grade C).

From the *McGill University Health Centre, Montréal, Que., the †Department of Urology, Queen's University, Kingston, Ont., the łDivision of Urology, Department of Surgery, University of Ottawa, Ottawa, Ont., the §Division of Urology, Centre Hospitalier Universitaire de Québec (CHUQ), Laval University, Laval, Que., the qDivision of Urology, Department of Surgical Oncology, Princess Margaret Hospital and the University Health Network, University of Toronto, Toronto, Ont., the **Division of Urology, Faculty of Medicine, University of British Columbia, Vancouver, BC, the ††Division of Urology, London Health Sciences Centre, University of Western Ontario, London, Ont., the $¥ \neq$ Department of Surgery, University of Alberta, Edmonton,

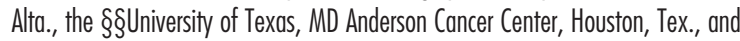
the ११Division of Urology, McGill University, Montréal, Que. 
Kassouf et al.

This article has been peer reviewed.

Competing interests: None declared.

\section{References}

1. Jemal A, Siegel R, Ward E, et al. Cancer statistics, 2006. CA Cancer I Clin 2006; 56:106-30.

2. Bergstrom $A$, Hsieh $C C$, Lindblad $P$, et al. Obesity and renal cell cancer - a quantitative review. Br J Cancer 2001;85:984-90.

3. Lindblad P. Epidemiology of renal cell carcinoma. Scand I Surg 2004;93:88-96.

4. Skolarikos $A$, Alivizatos $G$, Laguna $P$, et al. A review on follow-up strategies for renal cell carcinoma after nephrectomy. [discussion 1501]. Eur Urol 2007;51:1490-500.

5. Lau WK, Blute ML, Weaver AL, et al. Matched comparison of radical nephrectomy vs nephron-sparing surgery in patients with unilateral renal cell carcinoma and a normal contralateral kidney. Mayo Clin Proc 2000;75:1236-42.

6. Bani-Hani AH, Leibovich $B C$, Lohse $C M$, et al. Associations with contralateral recurrence following nephrectomy for renal cell carcinoma using a cohort of 2,352 patients. J Urol 2005;173:391-4.

7. Itano NB, Blute ML, Spotts B, et al. Outcome of isolated renal cell carcinoma fossa recurrence after nephrectomy. J Urol 2000;164:322-5.

8. Sandhu SS, Symes A, A'Hern R, et al. Surgical excision of isolated renal-bed recurrence after radical nephrectomy for renal cell carcinoma. BJU Int 2005;95:522-5.

9. Levy DA, Swanson DA, Slaton JW, et al. Timely delivery of biological therapy after cytoreductive nephrectomy in carefully selected patients with metastatic renal cell carcinoma. J Urol 1998;159:1168-73.

10. Lam JS, Shvarts 0, Leppert JT, et al. Renal cell carcinoma 2005: new frontiers in staging, prognostication and targeted molecular therapy. J Urol 2005;173:1853-62.

11. Janzen NK, Kim HL, Figlin RA, et al. Surveillance after radical or partial nephrectomy for localized renal cell carcinoma and management of recurrent disease. Urol Clin North Am 2003;30:843-52.

12. Liungberg B, Hanbury DC, Kuczyk MA, et al. Renal cell carcinoma guideline. Eur Urol 2007:51:1502-10.

13. Kim HL, Belldegrun AS, Freitas DG, et al. Paraneoplastic signs and symptoms of renal cell carcinoma: implications for prognosis. J Urol 2003;170:1742-6.

14. Kim HL, Han KR, Zisman A, et al. Cachexia-like symptoms predict a worse prognosis in localized t1 renal cell carcinoma. J Urol 2004;171:1810-3.

15. Patard JJ, Leray E, Cindolo L, et al. Multi-institutional validation of a symptom based classification for renal cell carcinoma. J Urol 2004;172:858-62.

16. Bensalah $\mathrm{K}$, Leray E, Fergelot $\mathrm{P}$, et al. Prognostic value of thrombocytosis in renal cell carcinoma. J Urol 2006; 175:859-63.

17. Patard JJ, Kim HL, Lam JS, et al. Use of the University of California Los Angeles integrated staging system to predict survival in renal cell carcinoma: an international multicenter study. I Clin Oncol 2004;22:3316-22.

18. Stephenson AJ, Chetner MP, Rourke K, et al. Guidelines for the surveillance of localized renal cell carcinoma based on the patterns of relapse after nephrectomy. J Urol 2004; 172:58-62.

19. Montie JE. Follow-up after partial or total nephrectomy for renal cell carcinoma. Urol Clin North Am 1994;21:589-92.

20. Sandock DS, Seftel AD, Resnick MI. A new protocol for the followup of renal cell carcinoma based on pathological stage. J Urol 1995;154:28-31.

21. Hafez KS, Novick AC, Campbell SC. Patterns of tumor recurrence and guidelines for followup after nephron sparing surgery for sporadic renal cell carcinoma. J Urol 1997; 157:2067-70

22. Canfield SE, Kamat AM, Sánchez-Oritz RF, et al. Renal cell carcinoma with nodal metastases in the absence of distant metastatic disease (clinical stage TxN1-2MO): the impact of aggressive surgical resection on patient outcome. J Urol 2006;175:864-9.

23. Saidi JA, Newhouse JH, Sawczuk IS. Radiologic follow-up of patients with TI-3a,b, or T4N+MO renal cell carcinoma after radical nephrectomy. Urology 1998;52: 1000-3.

24. Han KR, Pantuck AJ, Bui MH, et al. Number of metastatic sites rather than location dictates overall survival of patients with node-negative metastatic renal cell carcinoma. Urology 2003;61:314-9.

Correspondence: Dr. Wassim Kassouf, Division of Urology, McGill University, Department of Oncology, McGill University Health Centre, 1650 Cedar Ave., Montréal QC H3G 1A4; wassim.kassouf@muhc.mcgill.ca 